\title{
The Design of Structure and Simulation Analysis of Thermal Effect on Small Fiber Optic Gyroscope in Inclinometer While Drilling
}

\author{
Fan $\mathrm{Xu}^{\mathrm{a}}$, Jianhui Zhaob, Fan $\mathrm{Li}^{\mathrm{c}}$ and $\mathrm{He} \mathrm{Xu}^{\mathrm{d}}$ \\ School of Instrument Science and Opt-electronics Engineering, Beihang University, Beijing 100191, \\ China \\ ax123xufan@163.com, bzhaojianhui@buaa.edu.cn, clifan@buaa.edu.cn, dxuhe200907@163.com
}

Keywords: Quasi-elliptical cylindrical fiber optic gyroscope, Shupe effect, finite element, petroleum logging.

\begin{abstract}
A new quasi-elliptical cylindrical (QEC) fiber optic gyroscope (FOG) for petroleum logging is proposed for the special condition of the borehole diameter limitation in inclinometer while drilling (IWD). Due to the thermally induced nonreciprocal phase shift in fiber coil, the three-dimensional (3-D) temperature transient effects mathematical model and finite element model of QEC fiber coil are established by quadrupole (QAD) winding method, which are based on the Shupe effect of fiber coil and used to analyze the influence of structural parameters of QEC fiber coil on Shupe error. Combined with the actual working environment of FOG, the numerical simulation of QEC fiber coil is done to analyze the Shupe error in the fiber coil at certain temperature gradient quantitatively. It also verifies the correctness of model building comparing with the experimental results. The results show that when the fiber length, the number of fiber coil and the radius of fiber coil are fixed, the thermal error rate of QEC fiber coil is $40 \%$ to $50 \%$ smaller than that of FOG fiber coil.
\end{abstract}

\section{Introduction}

With the development of directional drilling technology, the measurement while drilling (MWD) based on FOG has become a hot spot in petroleum logging. However, the environment of petroleum logging is bad, such as the depth of deep well is several kilometers, the range of temperature in deep well varies greatly and the temperature rises by 3 degrees Celsius per 100 meters, and the size of IWD is limited by the borehole size ${ }^{[1]}$. Because of the FOG has the advantages of small size, high precision, low cost, free from geomagnetic interference, shock and vibration resistance, it becomes an ideal choice for near bit MWD in deep well and the compact space of metal casing ${ }^{[2]}$. However, there is temperature sensitivity in fiber coil, and the nonreciprocal phase shift caused by the uneven temperature field around leads the bias drift of FOG, which is known as the Shupe effect ${ }^{[3]}$. And the nonreciprocal error limits to improve the performance of FOG.

In order to reduce the influence of temperature, Mohr had established the temperature model of fiber coil, and it was concluded that reducing the length of fiber and increasing the number of winding layers could reduce the Shupe error by comparing experiments ${ }^{[4]}$. Frigo et al analyzed the Shupe effect quantitatively on the basis of the linear distribution of the temperature field, and proposed that the Shupe error was reduced by 3 orders of magnitude through the QAD ${ }^{[5]}$. Based on the numerical method, Tirat et al analyzed the temperature field in the fiber coil accurately by using IDEAS-TMG, which was the thermal analysis software ${ }^{[6]}$. Maochun Li proposed a 3-D temperature transient effects model to analyze the Shupe error caused by the axial asymmetric temperature gradient of the fiber coil sensitive axis ${ }^{[7]}$.

The above literatures are based on cylindrical FOG, and there is no analysis of thermally induced nonreciprocal effect in FOG under limited volume. Based on the above literatures, this paper proposes a new QEC fiber coil, and analyzes the influence of structural parameters of QEC fiber coil on Shupe error. The effectiveness of the proposed scheme is verified by theoretical analysis and simulation results, and the results show that the thermal error rate of QEC fiber coil is $40 \%$ to $50 \%$ smaller than that of FOG fiber coil under limited volume. 


\section{The physical model of QEC fiber coil}

The IWD is mainly composed of lower joint, outer cover, heat preserving barrel, phase changer body, FOG group and insulation plug. The design and installation of these components must be reasonable, which is closely related to the working accuracy of the IWD. Fig. 1 is the schematic diagram of IWD.

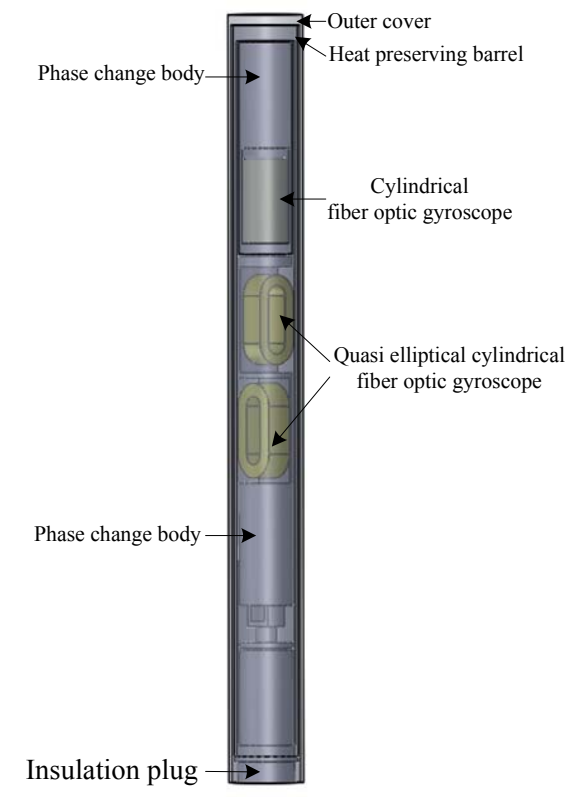

Fig. 1 The schematic diagram of IWD

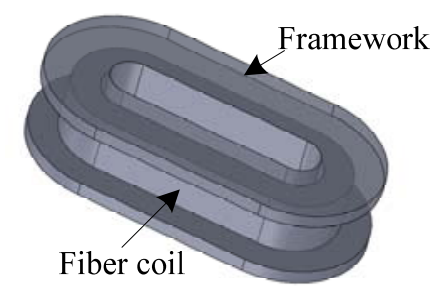

Fig. 2 The 3-D schematic diagram of QEC fiber coil

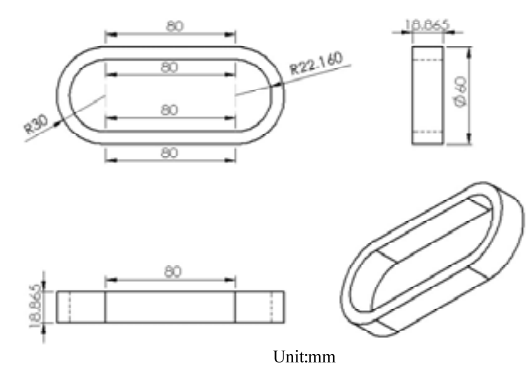

Fig. 3 The 2-D plane picture of QEC fiber coil

In the actual environment of petroleum logging, due to the small size of the borehole, the average diameter of IWD is only $60 \mathrm{~mm}$ or $80 \mathrm{~mm}$. However, the size of cylindrical FOG on the market is relatively large at present, which is limited in the application of IWD. The structure of FOG in IWD depends largely on the special structure of bottomhole assembly, and in order to ensure the normal working of IWD, a new QEC fiber coil is designed to improve the measuring accuracy of IWD, as shown in Fig. 2. Fig. 3 is the 2-D plane picture of QEC fiber coil. In the main view, there are semicircular in both ends of the QEC fiber coil, and the middle is connected by a straight line. The structure is slender in shape, which is fitted well into a cylindrical structure. 


\section{The 3-D Shupe error mathematical model of QEC fiber coil}

When the time-varying temperature perturbation occurs in fiber coil, the two beams of backward propagating light wave will undergo different phase shifts. The nonreciprocal phase shift caused by temperature and the Sagnac phase shift caused by rotation cannot be distinguished, which will generate bias error in FOG. There is the thermal error rate in formula(1).

$$
\Omega_{e}(t)=\frac{n}{4 A} \cdot \frac{\partial n}{\partial T} \cdot \int_{0}^{L} \dot{T}(s, t)(L-2 s) d s
$$

Where $n$ is the effective refractive index of fiber, $\partial n / \partial T$ is the temperature variation coefficient of refractive index, $L$ is the length of fiber, $A$ is the total effective area around the fiber coil, and $\dot{T}(s, t)$ is the rate change of temperature at time $t$ where is $s$ from the starting end. Formula (1) is established on the assumption that the temperature gradient is axially symmetric in the sensitive axis of the fiber coil and the temperature of the fiber coil is the same as that of the same layer, which can only simply analyze the thermally induced nonreciprocal effects caused by axial symmetric temperature gradients in the fiber coil sensitive axis. Therefore, it is necessary to establish a 3-D temperature transient effects mathematical model in fiber coil to analyze the thermally induced nonreciprocal effects caused by the radial, axial and circumferential temperature gradients.

The 3-D temperature transient effects mathematical model in fiber coil is based on the shape of fiber coil to select the coordinate system. Taking into account the special structure of QEC fiber coil, the cylindrical coordinate system and the Cartesian coordinate system are used to segment the integral, as shown in Fig. 4.

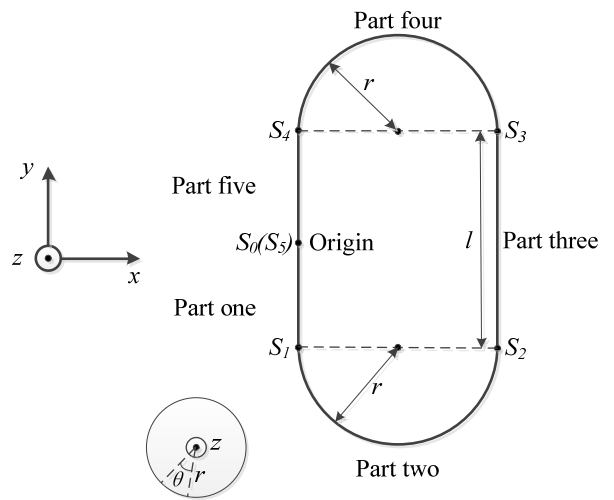

Fig. 4 The schematic diagram of integral process of QEC fiber coil

Where $r$ is the radius of the turn of fiber coil, $l$ is the length of the linear part, $S_{i}(i=1,2, \ldots, 5)$ is the distance from the point to the midpoint of fiber coil. The expression of the 3-D thermal error rate is obtained by where the midpoint of fiber coil is the origin $(s=0)$, the $s$ in counterclockwise $(\mathrm{CCW})$ is positive and the $s$ in clockwise $(\mathrm{CW})$ is negative.

$$
\begin{aligned}
& \Omega_{e}(t)=\frac{n}{2 A} \frac{\partial n}{\partial T} \cdot \\
& \left\{\begin{array}{l}
\sum_{i=1}^{N_{C C W}}\left\{\begin{array}{l}
\left.\int_{s_{i 0}}^{s_{i 1}} \frac{\partial \Delta T}{\partial t}\right|_{\left(x_{i}, y_{i}, z_{i}, t\right)} s d s+\left.\int_{0}^{\pi} \frac{\partial \Delta T}{\partial t}\right|_{\left(r_{i}, \theta, z_{i}, t\right)}\left(r_{i} \theta+s_{i 1}\right) r_{i} d \theta+\left.\int_{s_{i 2}}^{s_{i 3}} \frac{\partial \Delta T}{\partial t}\right|_{\left(x_{i}, y_{i}, z_{i}, t\right)} s d s \\
+\left.\int_{0}^{\pi} \frac{\partial \Delta T}{\partial t}\right|_{\left(r_{i}, \theta, z_{i}, t\right)}\left(r_{i} \theta+s_{i 3}\right) r_{i} d \theta+\left.\int_{s_{i 4}}^{s_{i 5}} \frac{\partial \Delta T}{\partial t}\right|_{\left(x_{i}, y_{i}, z_{i}, t\right)} s d s
\end{array}\right\} \\
-\sum_{j=1}^{N_{C W}}\left\{\begin{array}{l}
\left.\int_{s_{j 0}}^{s_{j 1}} \frac{\partial \Delta T}{\partial t}\right|_{\left(x_{j}, y_{j}, z_{j}, t\right)} s^{\prime} d s^{\prime}+\left.\int_{0}^{\pi} \frac{\partial \Delta T}{\partial t}\right|_{\left(r_{j}, \theta, z_{j}, t\right)}\left(r_{j} \theta+s_{j 1}\right) r_{j} d \theta+\left.\int_{s_{j 2}}^{s_{j 3}} \frac{\partial \Delta T}{\partial t}\right|_{\left(x_{j}, y_{j}, z_{j}, t\right)} s^{\prime} d s^{\prime} \\
+\left.\int_{0}^{\pi} \frac{\partial \Delta T}{\partial t}\right|_{\left(r_{j}, \theta, z_{j}, t\right)}\left(r_{j} \theta+s_{j 3}\right) r_{j} d \theta+\left.\int_{s_{j 4}}^{s_{j 5}} \frac{\partial \Delta T}{\partial t}\right|_{\left(x_{j}, y_{j}, z_{j}, t\right)} s^{\prime} d s^{\prime}
\end{array}\right\}
\end{array}\right\}
\end{aligned}
$$


Where $\partial \Delta T / \partial t$ is the rate change of temperature at time $t$ in a certain point of fiber coil, $N_{C C W}$ and $N_{C W}$ are the total turns of CCW and CW. Formula (2) shows that the thermal error rate induced by the temperature transient effects can be obtained by generating the rate change of temperature at any point in the fiber coil and accumulating the contribution of each turn fiber to the thermally nonreciprocal phase shift.

\section{The finite element model analysis of QEC fiber coil}

ANSYS is a general finite element computer program, which can analyze and calculate the problem of static, dynamic, thermal conduction, fluid mechanics and electromagnetic. The finite element model of QEC fiber coil is established by using ANSYS to solve the temperature field distribution information in the fiber coil based on temperature excitation. According the temperature distribution information of the QEC fiber coil, the temperature information needed by the 3-D Shupe error model can be obtained. The simulation parameters are shown in Table 1.

Table 1 The physical parameters of fiber coil

\begin{tabular}{cccc}
\hline Parameters & Density $\left(\mathrm{kg} / \mathrm{m}^{3}\right)$ & $\begin{array}{c}\text { Thermal Conductivity } \\
(\mathrm{W} /(\mathrm{m} \cdot \mathrm{K}))\end{array}$ & $\begin{array}{c}\text { Specific Heat } \\
(\mathrm{J} /(\mathrm{kg} \cdot \mathrm{K}))\end{array}$ \\
\hline Adhesive & 1000 & 0.08 & 2512 \\
Fiber & 1200 & 0.13 & 1302 \\
Composite & 1478 & 0.42 & 1280 \\
\hline
\end{tabular}

The model is based on QAD winding method, where the fiber length is $794 m$, the outer diameter of fiber is $245 \mu \mathrm{m}$, and the turns of each layer are the same. Fig. 5 is the temperature excitation applied to the outer ring of the fiber coil in the simulation. The temperature gradient of the temperature excitation is 1 degree Celsius per minute, which includes the heating stage and the cooling stage. Fig. 6 is the comparison of thermal error rate between the QEC fiber coil and the cylindrical fiber coil at a certain fiber length, where the inner diameter of the QEC fiber coil is $20 \mathrm{~mm}$, the layer of fiber coil is 32 , each layer is 80 turns, the length of straight linear part is $80 \mathrm{~mm}$, and the inner diameter of the cylindrical fiber coil is $20 \mathrm{~mm}$, the layer of fiber coil is 32 , each layer is 165 turns. Both QEC fiber coil and cylindrical fiber coil are using the same temperature excitation.

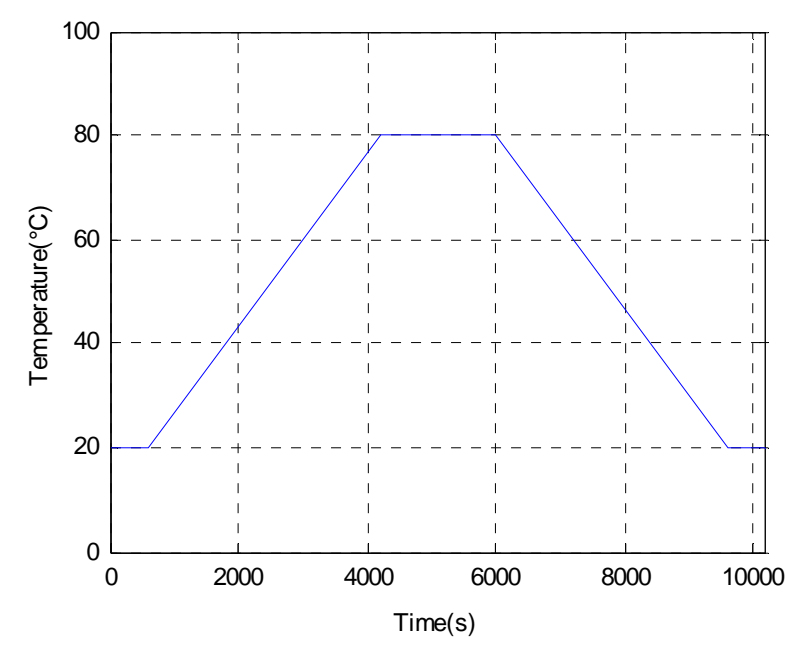

Fig. 5 The temperature excitation applied to the outer ring of fiber coil 


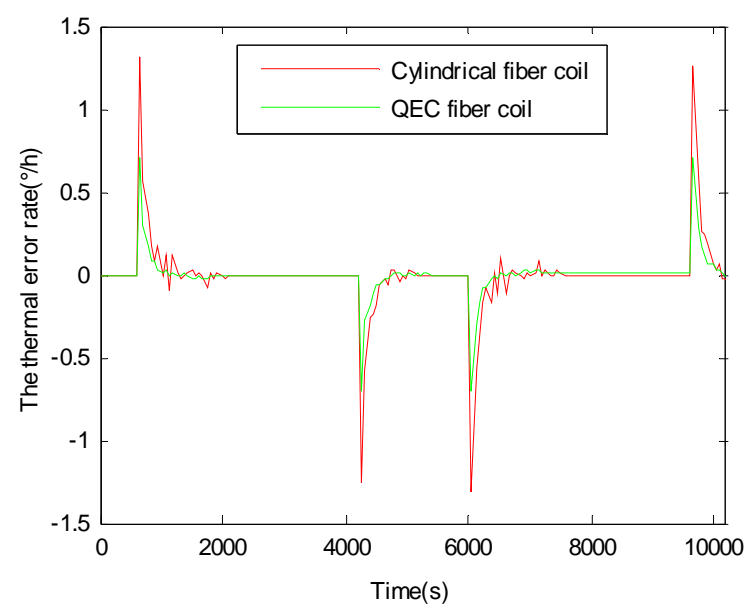

Fig. 6 The comparison of thermal error rate between the QEC fiber coil and the cylindrical fiber coil

It can be seen from the Fig. 6 that when the fiber length, the inner diameter and the layer of the fiber coil are fixed, the thermal error rate of the QEC fiber coil is small than that of the cylindrical fiber coil. In other words, the QEC fiber coil is better than the cylindrical fiber coil. With the fiber length, the inner diameter and the layer of fiber coil fixed, experiments are carried out by changing the corresponding structural parameters.

Table 2 The comparison between the QEC fiber coil and the cylindrical fiber coil

\begin{tabular}{ccccccc}
\hline Num. & Fiber coil & $\begin{array}{c}\text { Layer of } \\
\text { fiber coil }\end{array}$ & $\begin{array}{c}\text { Turns of } \\
\text { each layer }\end{array}$ & $\begin{array}{c}\text { Total effective } \\
\text { area }\left(\mathrm{m}^{2}\right)\end{array}$ & $\begin{array}{c}\text { The thermal } \\
\text { error rate }(\% / h)\end{array}$ & $\begin{array}{c}\text { Optimization } \\
\text { ratio }\end{array}$ \\
\hline \multirow{2}{*}{1} & Cylindrical & 24 & 230 & 9.1758 & 1.682 & \multirow{2}{*}{\begin{tabular}{c}
$4.5 \%$ \\
\multirow{2}{*}{2}
\end{tabular}} \\
& QEC & 24 & 109 & 13.9503 & 0.9678 & \\
& Cylindrical & 28 & 193 & 9.3864 & 1.553 & $46.6 \%$ \\
3 & QEC & 28 & 92 & 14.1312 & 0.8288 & \\
& Cylindrical & 32 & 165 & 9.5758 & 1.306 & \multirow{2}{*}{$46.6 \%$} \\
\hline
\end{tabular}

It can be seen from the Table 2 that when the fiber length, the inner diameter and the layer of the fiber coil are fixed, due to the fiber length of each turn in QEC fiber coil is larger than that in cylindrical fiber coil, the turns per layer of QEC fiber coil is smaller than that of cylindrical fiber coil. In addition, the total effective area of the QEC fiber coil is larger than that of the cylindrical fiber coil. The sensitivity of the FOG is only related to the total effective area of the fiber coil, and it is proportional to the total effective area. As can be seen from the Table 2, the thermal error rate of QEC fiber coil is $40 \%$ to $50 \%$ smaller than that of cylindrical fiber coil. Based on the above analysis, we can draw the conclusion that with the fiber length, the inner diameter and the layer of fiber coil fixed, the QEC fiber coil is better than the cylindrical fiber coil when the temperature excitation is applied on the outer ring of the fiber coil.

\section{Structural optimization and quantitative design}

Shupe error is affected by the structural parameters of the fiber coil, the structural parameters include the inner diameter of fiber coil $(R)$, the length of the linear part $(l)$, the length of fiber $(L)$, the number of fiber coil layers $(N)$ and the number of turns per layer $(M)$. On the one hand, the structure parameters affects the calculation of thermal error rate, on the other hand, it affects the temperature distribution in the fiber coil.

\subsection{Analysis of the influence of inner of fiber coil on thermal error rate of QEC fiber coil.}

With the fiber length $(L=794 \mathrm{~m})$, the length of linear part $(l=80 \mathrm{~mm})$ and the layer of fiber coil fixed, simulation experiments are carried out to get the thermal error rate of QEC fiber coil by changing the inner diameter of fiber coil, as shown in Fig. 7. 


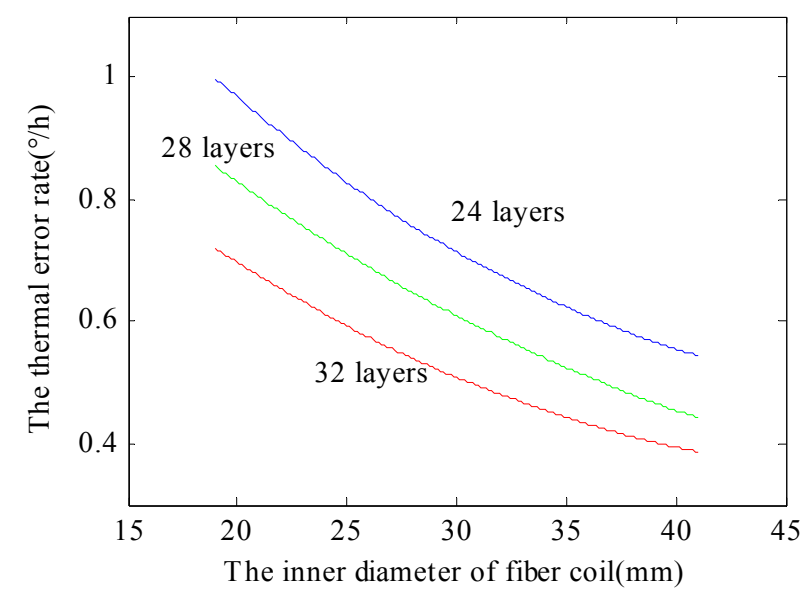

Fig. 7 The thermal error rate at different inner diameter of QEC fiber coil

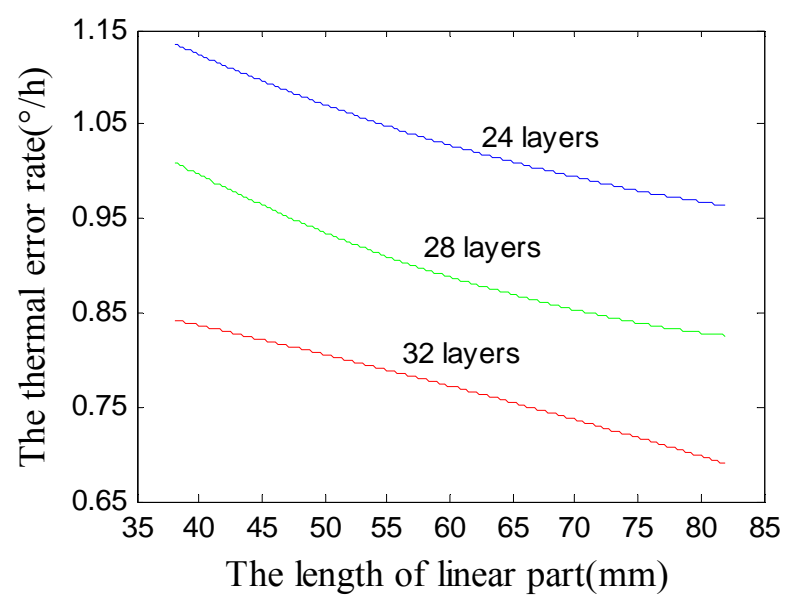

Fig. 8 The thermal error rate at different length of linear part of QEC fiber coil

Under the condition that the length of fiber is certain and the layer of fiber coil is the same, if the inner diameter of fiber coil increases, then the number of turns of each layer decreases as well as the length difference of adjacent layer fiber also decreases. It can be seen from the Fig. 7 that the thermal error rate of fiber coil decreases approximately with the increase of the inner diameter of fiber coil when the layer of fiber coil is fixed, which shows that increasing the inner diameter of fiber coil helps to reduce the thermal error rate. When the inner diameter of the fiber coil is fixed, the thermal error rate of fiber coil decreases with the increase of the layer of fiber coil, and the turns of each layer decreases accordingly. It shows that increasing the layer of fiber coil or reducing the turns of each layer helps to reduce the thermal error rate.

5.2 Analysis of the influence of the linear part length on thermal error rate of QEC fiber coil.

With the fiber length $(L=794 \mathrm{~m})$, the inner diameter of fiber coil $(R=20 \mathrm{~mm})$ and the layer of fiber coil fixed, simulation experiments are carried out to get the thermal error rate of QEC fiber coil by changing the length of linear part, as shown in Fig. 8.

Under the condition that the length of fiber is certain and the layer of fiber coil is the same, the turns of each layer decreases with the increase of the length of linear part. It can be seen from the Fig. 8 that the thermal error rate of fiber coil decreases approximately with the increase of the length of linear part when the layer of fiber coil is fixed, which shows that increasing the length of linear part of fiber coil helps to reduce the thermal error rate. When the length of linear part of the fiber coil is fixed, the thermal error rate of fiber coil decreases with the increase of the layer of fiber coil, and the turns of each layer decreases accordingly. 
5.3 Analysis of the influence of the fiber length on thermal error rate of QEC fiber coil.

Table 3 The thermal error rate at different fiber length of QEC fiber coil

\begin{tabular}{cccccc}
\hline Num. & $\begin{array}{c}\text { Layer of } \\
\text { fiber coil }\end{array}$ & $\begin{array}{c}\text { Turns of } \\
\text { each layer }\end{array}$ & Length of fiber $(m)$ & $\begin{array}{c}\text { Total effective } \\
\text { area }\left(m^{2}\right)\end{array}$ & $\begin{array}{c}\text { The thermal } \\
\text { error rate }(\% / h)\end{array}$ \\
\hline 1 & 24 & 109 & 796 & 13.9503 & 0.9678 \\
2 & 24 & 130 & 949 & 16.6380 & 1.17 \\
3 & 24 & 150 & 1095 & 19.1976 & 1.362 \\
\hline
\end{tabular}

Simulation experiments are carried out to get the thermal error rate of QEC fiber coil by changing the turns of each layer of fiber coil when the inner diameter, the length of linear part and the layer of fiber coil are fixed, as shown in Table 3. It can be seen that under the condition that the inner diameter and the length of linear part of fiber coil are fixed, the thermal error rate of QEC fiber coil increases with the increase of the fiber length. However, increasing the length of fiber can increase the effective area of fiber coil, thus increasing the sensitivity of the fiber coil, there are conflict between sensitivity and thermal error rate.

\section{Summary}

From the above simulation results, we can draw conclusions that the QEC fiber coil is better than the cylindrical fiber coil, and in order to reduce the thermal error rate of QEC fiber coil, it is necessary to adopt a larger diameter and larger linear part length fiber coil, the layer of fiber coil and the turns of each layer should be reasonable, and improve the thermal conductivity of fiber coil.

\section{References}

[1] Seibi A., Karrech A., Boukadi F., et al. Wellbore Path Estimation Using Measurement While Drilling Techniques: A Comparative Study and Suggestions for Improvements. Energy Sources, Part A: Recovery, Utilization, and Environmental Effects. Vol. 31 (2009), p. 1205-1216.

[2] Rong Li, Jianhui Zhao, Fan Li. On gyroscopic MWD based on axial velocity integrated method. Well Logging Technology. Vol. 36(2012), p. 192-196.

[3] Shupe D. M. Thermally Induced Nonreciprocity in the Fiber Optic Interferometers. Applied Optics. Vol. 19(1980) No. 5, p. 654-655.

[4] Mohr F. Thermo optically induced bias drift in fiber optical Sagnac interferometers. Journal of Light wave Technology. Vol. 14(1996) No. 1, p. 27-41.

[5] Frigo N. J. Compensation of linear sources of non-reciprocity in Sagnac interferometers. SPIE Proceedings. Vol. 412(1983), p. 268-271.

[6] Tirat O. F. J. Finite element model of thermal transient effect in fiber optic gyro. SPIE Proceedings. Vol. 2837(1996), p. 230-238.

[7] Maochun Li, Tiegen Liu, JiaJia Wang, et al. Three-dimensional finite element model of Shupe effect in FOG fiber coil. Journal of Tianjin University. Vol. 41(2008) No. 5, p. 601-606. 